\title{
Investigación humanística en América Latina: experiencias con comunidades indígenas
}

Dra. Deborah Leal Rodriguesi y Dra. Heidy Vega Garcíai, Universidad Nacional

Recibido: 14-8-2014

Aprobado: 19-11-2014

\section{Resumen}

Este artículo resume el trabajo de investigación y extensión de dos académicas del Centro de Estudios Generales (CEG) de la Universidad Nacional de Costa Rica, entre los años 2007 y 2014. Desde la perspectiva del Nuevo Humanismo, se destaca la relevancia que la metodología participativa tiene para la apertura de líneas de diálogo y trabajo solidario con las comunidades indígenas de América Latina, como el desarrollado específicamente por el Proyecto Plataforma DESC ARUANDA.

\section{Abstract}

\section{Humanistic Research in Latin America: Experiences with Indigenous Communities}

This article summarizes the outreach/research work of two scholars of the Center of Humanities Studies (Centro de Estudios Generales) of the Universidad Nacional de Costa Rica, between the years 2007 and 2014. From the perspective of the New Humanism, the relevance of the participatory methodology has for opening new lines of dialogue and solidary work in indigenous communities in Latin America is stressed, as the one specifically performed by the Platform Project (Proyecto Plataforma) DESC ARUANDA.

i Deborah Leal es Doctora en Estudios Latinoamericanos con Mención en Pensamiento Latinoamericano, graduada de la Universidad Nacional de Costa Rica. Tiene además un Magister en Agroforestería Tropical, del CATIE, en Costa Rica y una licenciatura en Medicina Veterinaria, obtenido en la Facultad de Ciencias Agrarias de Pará, Brasil. Labora como investigadora del Área de Ciencia y Tecnología del Centro de Estudios Generales, Universidad Nacional de Costa Rica. Ha publicado numerosos y destacados estudios y ponencias en el área de su interés.

ii Heidy Vega es Doctora en Estudios Latinoamericanos con Mención en Pensamiento Latinoamericano, graduada de la Universidad Nacional de Costa Rica. También tiene el grado Magister en Relaciones Internacionales y en Diplomacia con Mención en Ambiente y Desarrollo, obtenidos ambos posgrados en esa misma casa de estudios. Es académica destacada, investigadora del Area de Ciencia y Tecnología del Centro de Estudios Generales, UNA y autora de varios artículos y ponencias publicados internacional y nacionalmente.
Deborah Leal Rodrigues y Heidy Vega García. Investigación humanística en América Latina: experiencias con comunidades indígenas. Revista Comunicación. Año 35, vol. 23, núm. 2. Julio-diciembre, 2014. Tecnológico de Costa Rica. ISSN impreso: 0379-3974/ e-ISNN: 1659-3820

PALABRAS CLAVE:

humanismo, investigación, metodología, pueblos indígenas, Latinoamérica.

KEY WORDS:

Humanism, Research, Methodology, Indigenous Peoples, Latin America. 


\section{INVESTIGAR EN HUMANIDADES}

La comunidad científica debe aceptar que el pensamiento vivo también es "no académico". Por lo tanto, en América Latina es necesario reivindicar las otras formas de pensar que existen, ya que nuestra región posee una historia cultural particular con diversas formas de comprender la realidad. La capacidad de "pensar" no es una exclusividad del pensamiento europeo occidental. Es necesario alzar la voz y dar a conocer al mundo las ideas, los conceptos, las cosmovisiones de las sociedades tradicionales. La academia latinoamericana debe promover una mayor amplitud de conciencia, un verdadero conocimiento interior y una integración de los diferentes aspectos del ser latinoamericano. Es necesario profundizar la dimensión práctica del conocimiento, que no debe ser solamente teoría desapegada de la realidad, sino que debe convertirse en la clave para resolver las crisis actuales de ignorancia, pobreza, corrupción, indiferencia, egoísmo, maldad y destrucción humana y planetaria.

Desde la perspectiva metodológica, las humanidades tienen la tarea de investigar y accionar, trabajar con gente real, con procesos y realidades concretas y provocar cambios concretos en estas realidades. En la interacción con las comunidades indígenas, es necesaria la reflexión y sistematización de las experiencias, perspectivas, metodologías, recursos y diversas actividades desarrolladas en la investigación, por medio de una relectura "humanista" de las realidades y vidas locales latinoamericanas, sus culturas y sus contextos. Por lo tanto, las metodologías de investigación-acción participativa en comunidades indígenas de América Latina deben abordar aspectos como la cosmovisión, la espiritualidad, la identidad, los sistemas de valores, las dinámicas comunitarias, el lenguaje simbólico y los sistemas adaptativos locales, que son determinantes para que la investigación humanista sea una experiencia de vida más allá de lo estrictamente académico.

El proceso de trabajo del CEG ha evolucionado como expresión de un Nuevo Humanismo Latinoamericano. Por esto, uno de los temas de mayor interés que se ha venido incorporando de lleno en el contenido curricular de los cursos que se imparte y en las investigaciones que se realiza es el tema de los pueblos indígenas ${ }^{1}$. Este interés surge ante la necesidad de sensibilizar, rescatar, resignificar, socializar y respetar la diversidad cultural latinoamericana para que los futuros profesionales y líderes (indígenas y no indígenas) puedan construir una sociedad más justa e inclusiva para todos.
La investigación-acción nace con procesos de autoconocimiento, forjados entre actores y movimientos sociales, frente al hecho ineludible de desarrollar metodologías más afines a la realidad local "para provocar y apoyar cambios estructurales y puntuales". Investigar y accionar es trabajar con gente real, con procesos y realidades concretas y es provocar cambios concretos en estas realidades. La investigación-acción no es necesariamente la más precisa: su rigurosidad se relaciona con precisiones necesarias en coyunturas específicas, recursos humanos y materiales, además de que busca ser sostenible por sí misma, en el balance entre esfuerzos y profundidades. Nace cuando los actores descubren que nadie investigará sus problemas particulares, dada su complejidad y singularidad. Las experiencias en autoconocimiento representan la máxima expresión de la investigación-acción en comunidades, que, desde una visión compleja, viene acopiando, rescatando y reconstruyendo métodos ancestrales y contemporáneos. La reconstrucción se apoya desde diferentes ejes temáticos; se fortalece el pensamiento y la capacidad de comprensión de la realidad, y se favorecen reconstrucciones en diferentes campos, como salud, agricultura, infraestructura y desarrollo sostenible en general. Los actores han desarrollado herramientas diversas y creativas en el abordaje y comprensión de sus realidades. El desarrollo de las herramientas obedece, primero, a reposicionamientos políticos y teóricos elementales, y segundo, a la transdisciplinariedad de los investigadores. La lectura de las realidades provoca el desarrollo de estrategias de entrada y comprensión y de fusión de variables desvinculadas, en el contexto de la investigación convencional. De la sensibilidad de cada uno y de sus experiencias e intereses únicos, nacen nuevos enfoques, herramientas y procesos en los diferentes campos de la realidad.

\section{MODELO CONCEPTUAL}

De acuerdo con Vega (2013), el tejido es una de las principales expresiones artísticas de las culturas ancestrales latinoamericanas y por tanto, se parte del "modelo de análisis de la metáfora del tejido" para explicar el conjunto de la relación holística de las categorías de análisis empleadas. Cada una de estas categorías representa un hilo, que al unirse con el resto de los hilos (las otras categorías) forma una red (trama, urdimbre) que le da sentido al conjunto. Para comprender el tejido, hay que mirar cada hilo que lo conforma, ver cómo se relaciona con los demás, al igual que en nuestra vida, la visión de mundo, los hábitos, las costumbres, los gustos y las actividades, dependen de 
una relación interdependiente de factores. Así, de la unión de todos los hilos se crea un panorama integrador del tema investigado.

Figura 1

Categorías de análisis empleadas en la investigación

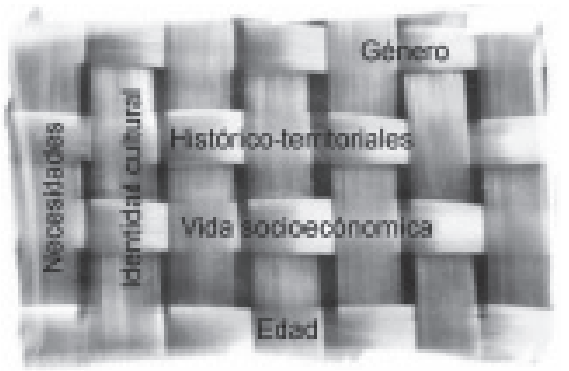

Fuente: Vega (2013)

Como punto de partida, Vega (2013) identifica la existencia de tres tipos de hilos fundamentales:

a. Los factores de base histórico-territorial: son aqueIlos que, por su propia naturaleza, son los más permanentes en el tiempo y los que definen la realidad material de los actores que se desarrollan en un territorio específico. Entre ellos destaca la historia y las características ambientales del entorno en el que se nace y se crece.

b. Los factores de identidad cultural: caracterizados por definir una visión interpretativa sobre la realidad material, a partir de valores culturales compartidos en una comunidad. En este sentido, es necesario considerar el grupo étnico al que se pertenece, los valores espirituales, familiares y sociales que se aprenden, se practican y se comparten, y los símbolos con los cuales se identifica.

c. Los factores intra-sociales: se trata de aquellos relacionados con el desarrollo de prácticas de vida socioeconómica derivadas de los anteriores factores mencionados. Entre ellos destaca el género, la edad, el nivel educativo, ocupacional, etc.

Para efectos de la especificidad de la investigación, se puede destacar un factor intra-social específico, como por ejemplo el de la perspectiva de género.

Finalmente, en este análisis también deben incluirse los factores externos (planetarios), los cuales determinan la interrelación entre los factores coyunturales de base histórico-territorial, los factores de identidad cultural y los factores intra-sociales de un lugar específico, con los problemas y dinámicas globales. Para este caso específico, se puede estudiar la influencia del contexto planetario en lo local, así como la trascendencia de lo local hacia lo planetario, para que la unión del conjunto de todos ellos sustente un tejido compacto. Es importante considerar que se encuentran sujetos a dinámicas de permanencia y adaptabilidad en el tiempo.

Figura 2

Tejido maya elaborado por una artesana kakchiquel

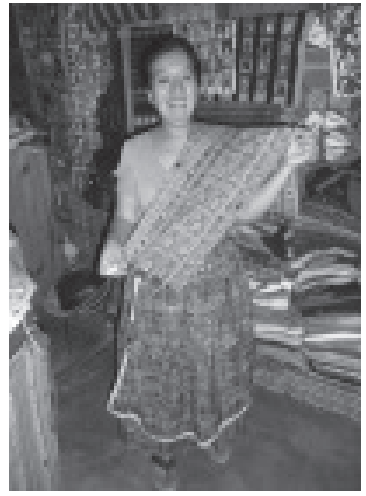

Fuente: Vega (2013) Trabajo de campo, marzo 2012

\section{METODOLOGÍAS DE INVESTIGACIÓN-ACCIÓN PARTICIPATIVAS EN COMUNIDADES INDÍGENAS DE AMÉRICA LATINA}

La metodología participativa (cualitativa) de tipo descriptiva-explicativa es la encargada de dar unidad e integralidad a los procesos investigativos, y de brindar sentido y significado para la vida (Abarca, 2013). Por tanto, la metodología participativa permite acercarse al paradigma holístico y de la complejidad y concibe la realidad como integral, interdependiente, capaz de poner la vida como el centro en conectividad con todos los seres vivos y la naturaleza.

Esta metodología se fundamenta en los aspectos epistemológicos referentes a la integralidad y complejidad de la vida, la naturaleza y las personas como interdependientes, diversas, históricas, cambiantes y contradictorias. Si la vida está constituida por procesos, sistemas, ondas, fluctuaciones, complejidad, incertidumbre y caos, para que la investigación tenga sentido se necesita una concepción epistemológica y metodológica capaz de armonizar todo lo anterior pero al mismo tiempo, de convertir los procesos de investigación en importantes espacios para la reflexión, la construcción colectiva de conocimientos y la 
elaboración de propuestas y acciones conjuntas para el mejoramiento de las condiciones de vida.

Abarca (2013) también destaca que la metodología participativa recupera lo "no visible": afectos, subjetividad, percepciones, intuiciones y sentidos a partir de la integralidad de las dimensiones investigativa, pedagógica, comunicativa y creadora (productiva), que remite a la interdependencia de nuestras acciones en los procesos de intervención social.

La dimensión investigativa por su parte, está siempre presente en los procesos de intervención social y tiene como momento culminante el proceso de construcción de conocimientos y la búsqueda y recuperación de la memoria colectiva, de los hallazgos contextuales y de las formas particulares en que las personas sienten, perciben e interpretan determinados fenómenos. De igual modo, busca diversas fuentes y estudios especializados existentes para profundizar las discusiones y elaboraciones teóricas en los procesos de intervención social.

De igual modo, la dimensión pedagógica orienta el tránsito ordenado y sucesivo de conocimientos sobre algunos fenómenos -sociales o naturales- hacia la comprensión teórica global de esos procesos educativos vinculados con la vida, porque aprender es vivir.

Por su lado, la dimensión comunicativa está constantemente presente para permitir nuestros diálogos creadores, el intercambio de conocimientos, reflexiones, opiniones y conclusiones, la promoción de los interaprendizajes y el enriquecimiento permanente del propio saber con el de otras personas, involucrando nuestra participación en bucles de realimentación permanentes.

Finalmente, la dimensión creadora está presente en todo el proceso y no como producto final, ya que ambos elementos son generados en el momento en que se generan acciones en que dinamizan y realimentan el proceso. El objetivo viene a ser lograr que las personas en interacción construyan la expresión creadora que trasciende y realimenta al mismo tiempo.

En el quehacer académico se prioriza una de estas dimensiones, pero siempre se aplican las otras: siempre se investiga, se aprende, se comunica y se elaboran productos creativos; incluso al utilizar las vivencias personales se ponemos en práctica el bucle o rizo metodológico de la relación Práctica-Teoría-Práctica（Leis, 1989).

Según Leis (1989), esta relación Práctica - Teoría - Práctica tiene su fundamento en el hecho de que la práctica social es fuente, criterio de verdad y fin últi- mo del proceso de conocimiento pero también trata del conjunto de actividades hechas consciente e intencionalmente en las áreas económicas, políticas, ideológicas, culturales y hasta en las cotidianas. Por eso, la reflexión que se haga sobre esta práctica y esta realidad no pueden ser ideas abstractas, sino que deben confrontarse continuamente con la realidad y la práctica pues la finalidad de todo este proceso de conocimiento es la transformación de la realidad. Para Abarca (2013), la aplicación del rizo o bucle metodológico implica la construcción de un proceso integral de conocimiento, sustentado en la realización de tres momentos conectados entre sí e interdependientes:

a. Primer momento (práctica): Se parte del contexto, las problemáticas, las necesidades, los saberes, los sentimientos, las experiencias, los conocimientos, las interculturalidades y las tradiciones propias.

b. Segundo momento (teoría): Se reflexiona, se conceptualiza y se construye el conocimiento de forma colectiva.

c. Tercer momento (práctica): La vuelta a la práctica, donde se aplican los nuevos conocimientos al contexto, a la vida cotidiana, a la realidad en que actuamos, en busca del cambio de las condiciones de vida.

En las investigaciones realizadas por quienes escriben, se ha pretendido revelar los significados que sustentan las acciones e interacciones de la realidad social de los pueblos indígenas. De vuelta a la práctica, en la investigación se ha procurado, además de generar conocimiento, impulsar la transformación de la realidad latinoamericana y planetaria, mediante la difusión de valores para la Vida, inspirados por la cosmovisión y las prácticas socioambientales de los pueblos indígenas de América Latina.

\section{MÉTODOS Y TÉCNICAS PARTICIPATIVAS, RECURSOS Y ACTIVIDADES DIVERSAS DESARROLLADAS POR LAS INVESTIGADORAS}

Además de la recolección de información, mediante la revisión documental y de otras fuentes (audiovisuales, por ejemplo), se destaca la vivencia in situ de experiencias con las comunidades de interés, como el principal insumo obtenido del trabajo de campo. Este resulta fundamental, ya que la convivencia con las personas y grupos de estudio de forma directa amplía el conocimiento de las características ambientales, socioeconómicas y culturales del entorno, las cuales inciden la visión de 
mundo de las comunidades, tanto en sus dinámicas sociales como en las expectativas individuales.

De forma particular, en las investigaciones se han aplicado métodos y técnicas participativas, como la observación participante, el registro escrito de imágenes y notas de campo, las entrevistas semiestructuradas, las conversaciones colectivas y la participación directa en actividades concretas de trabajo comunitario, por ejemplo, en el quehacer de las cooperativas. Además, se ha recurrido al registro audiovisual de testimonios e historias de vida, con el fin de recuperar la historia y cultura de la memoria colectiva de cada comunidad y los valores ancestrales de las culturas ancestrales.

\section{INTERACCIÓN CON LAS COMUNIDADES INDÍGENAS: PLATAFORMA DESC-ARUANDA DEL CENTRO DE ESTUDIOS GENERALES (CEG)}

Desde el 2008 se han realizado diferentes estudios comunitarios transversales e investigación - acción participativa, con el fin de cumplir los derechos económicos, sociales y culturales de las comunidades (DESC).

Es de destacar que entre 2008-2012 se realizó un diagnóstico DESC en los territorios indígenas de Costa Rica y entre los principales resultados de este período, se determinó no solo un panorama de aislamiento relativo en las diferentes comunidades sino también de resistencia local no organizada a la interminable invasión de sus territorios y expansión de comercios y fincas de no indígenas; también se logró la búsqueda propia de alternativas de sobrevivencia afuera de sus territorios ancestrales y se confirmó que un 50\% de los indígenas de Costa Rica hoy viven (aunque temporalmente) en las principales ciudades del país (ARUANDA, 2012).

Actualmente, el rescate de las cosmovisiones ancestrales comprende la reunión de los fragmentos visibles dentro de las culturas indígenas contemporáneas, a partir de la reconstrucción de los elementos y marcos más amplios de las culturas, de su historia de exterminio y sobrevivencia, de los testimonios de las personas de conocimiento y de la recopilación de sus memorias, mitos, historias, lenguas y prácticas cotidianas de sobrevivencia. Además, ARUANDA desarrolla un modelo de trabajo comunitario que coordina las actividades académicas junto con los cursos impartidos en el CEG/ UNA. El proyecto tiene como meta de largo plazo generar información pertinente para la preservación de la memoria cultural de los pueblos autóctonos del planeta e incentivar nuevos programas de estudios humanísticos, enfocados en el Buen Vivir, los DESC y la recons- trucción cultural y de la vida como un todo (ARUANDA, 2013). Este proyecto del CEG ha funcionado como un espacio para favorecer el diálogo entre los indígenas y su entorno. Entonces, mediante diversas actividades de experiencias en investigación-acción participativa en comunidades indígenas de Costa Rica (con estudiantes y actores comunitarios) se ha promovido el autodiagnóstico, la planificación, la sistematización y la divulgación sobre los derechos económicos, sociales y culturales de los pueblos indígenas. Este proyecto procura también la construcción de procesos educativos emancipadores, orientados a la formación de masas críticas, capaces de entender "el lenguaje del otro" y apropiarse, crítica y selectivamente, de sus recursos y capacidades. Estas masas críticas, al empoderarse de sus procesos, podrán delimitar sus territorios de acción y de vida y, en consecuencia, decidirán cómo y en qué relacionarse "con el otro", desde un posicionamiento congruente con los intereses de su grupo, incidiendo así en la "otra sociedad". Lo propuesto enfoca la formación de profesionales interculturales, desde una perspectiva de construcción pedagógica, trabajada como un desafío por superar las limitaciones de los paradigmas fragmentarios occidentales, hacia la estructuración de paradigmas interculturales de aprendizaje e investigación, abarcadores de la complejidad de las realidades locales y globales (Leal, 2007).

En la ruta de observación y comprensión del mundo de las culturas indígenas, se encontraron alternativas de reconstrucción: todo es comunicación o pasa por ella. Además, los procesos de autodeterminación dependen de la comunicación que se desarrolla en diferentes niveles: entre personas, organizaciones, países y regiones, con problemas o características comunes. La comunicación se extiende al reconocimiento geográfico, en los mapas étnicos, en los mapas de los recursos naturales, en el arte, la artesanía, todos los cuales son también instrumentos de comunicación y mapas indescifrables para otras culturas (Leal, 2007).

La palabra también es un mapa que acerca o aísla, de manera que la estructura de los discursos es fundamental en el proceso de comunicación. El discurso construye puentes, palabras de contacto que hacen más eficiente el diálogo, o acercan iguales o más iguales. En esto se basa el diálogo de saberes: en la transformación en diálogo entre los más parecidos en medio de la diversidad, de manera el diálogo de saberes acerque verdades más comprensibles entre actores más cercanos (Leal, 2007). 
En los procesos de reconstrucción, es importante considerar un acercamiento respetuoso a la cultura, relaciones y roles de poder dentro de las comunidades y fuera de ellas. Por eso, la observación cuidadosa de las propuestas educativas y de reconstrucción cultural, el tratamiento del tema educativo y el desarrollo de enfoques integrados de valores políticos, institucionales, académicos y económicos en los proyectos educativos, resultan la primera premisa para el acercamiento a las realidades y a los procesos locales. Recuérdese que el abordaje de las culturas e intereses de los grupos, como complejidades que son, deben ser producto de la cosmovisión de individuos, familias extendidas y del clan, como un todo.

Posteriormente, se deben considerar las relaciones políticas de los grupos con la sociedad local y la política nacional, lo cual, incluye conocer y reconocer los espacios de representación conquistados, el grado de empoderamiento de sus procesos y su incidencia frente a la sociedad dominante (Leal, 2007). En este punto, es importante estimar las necesidades de las culturas y asumirlas como asuntos cambiantes en el tiempo. Por esto, el enfoque de ARUANDA en las comunidades es transversal en el tiempo y parte del cumplimiento de sus DESC.

Las observaciones, igualmente, deben trascender tanto las relaciones de los grupos con el mercado y la sociedad como las ventajas logradas entre ellas. Es entonces cuando las acciones pedagógicas para la autosostenibilidad de las comunidades se remiten al estudio y reproducción de los sistemas adaptativos y tecnologías relacionadas con el manejo de los recursos naturales y de la cultura, desde un enfoque también planteado por ARUANDA como transdisciplinario e intercultural. Empero, siempre es importante visualizar la articulación entre personas, familias, comunidades y culturas, así como las relaciones con los actores socioculturales externos (Leal, 2007).

Ahora bien, resulta fundamental rescatar y reconocer las iniciativas educativas anteriores, implementadas para entender la dinámica de lo que "podría funcionar" con estos colectivos, pues esos diagnósticos iniciales permitirían establecer las premisas básicas y las estrategias para el desarrollo de programas educativos apropiados a las realidades comunitarias. En ese contexto, no pueden dejar de reconocerse las necesidades y condiciones de los actores de tomar cursos universitarios de duración ampliada, ya que el análisis del contexto local es siempre el punto de partida de cualquier iniciativa de ARUANDA. En este caso específico, se debe considerar la posibilidad de trabajar primeramente con la educación primaria y secundaria, a sabiendas de que su resultado es de largo plazo y que el tiempo de estos pueblos frente a la convivencia con la cultura dominante, no es eterno. Valga anotar que es en esta etapa en la que se deben reconocer los apoyos institucionales, políticos y económicos que se podrán lograr para iniciar los proyectos educativos, en concordancia con la cronología de las acciones educativas y el cumplimiento de los DESC (Leal, 2007).

Por otra parte, se ha observado que la construcción sostenible de procesos educativos demanda que se trabaje en ciertos ejes como:

a. Institucional - administrativo: pensado desde estructuras móviles, poco dispendiosas y abiertas a alianzas con otras instituciones.

b. Político: específicamente, cómo buscar la independencia entre el proceso académico y el proceso político ${ }^{2}$.

c. Relacional - comunitario, que involucra no separarse de las comunidades y de los dirigentes políticos, por medio del diálogo permanente con los grupos y la delegación de funciones y promoción de centros de estudios en las comunidades como termómetros de los intereses comunitarios.

d. Académico: sin olvidar que su primera necesidad consiste en formar profesionales para que formen a otros.

e. Económico: recuérdese cuán importante es buscar la sostenibilidad de la iniciativa educativa, mediante el autofinanciamiento progresivo de las actividades (Leal, 2007).

En este sentido, los proyectos pioneros se multiplicarán en planes de estudios apropiados, con énfasis en la investigación y en el espíritu emprendedor de los estudiantes. Esos procesos deberán acompañarse de un desarrollo metodológico especializado en el tratamiento y sistematización de realidades. Así las cosas, una estrategia eficaz para lograr el éxito sería contar con profesionales en diferentes áreas del conocimiento (biología, sociológica, pedagogía, conocimientos ancestrales), quienes serían los responsables de orientar y conducir la investigación, sistematización y el desarrollo de los planes de estudio.

Igual es de hacer notar que la investigación en comunidades debería estar orientada por los estudiantes 
y personas con conocimientos tradicionales y las metodologías basarse en fenómenos y no disciplinas. Las acciones para el "Buen vivir" se condensarían en los procesos educativos en comunidades (Leal, 2007).

Aquí se propone la reconstrucción de la paz en el conflicto (Iglesias Díaz, 1999, p. 23), mediante la formación e información de diferentes actores sociales, entre ellos ancianos, mujeres, hombres, niños, jóvenes, autoridades y formas de organización, representación y expresión tradicionales y modernas. Se asume además, que la paz se construirá a partir del desarrollo del diálogo intercultural para el conocimiento del "otro" y la construcción de afinidades, mediante la convivencia en nuevos contextos, sustentada en el reconocimiento de los límites de las otras culturas y de su propia cultura (Leal, 2007).

Ahora bien, es importante rescatar que una construcción de una educación empoderadora para las colectividades, como la que se desea aquí, ocurre con la resignificación de contextos y el desarrollo de posicionamientos que fortalezcan la autodeterminación, la planificación participativa de programas de estudios aptos a las realidades, el desarrollo de métodos con sus niveles generativos y estratégicos y de herramientas de enseñanza y de sistematización eficaces para atender a las realidades, el desarrollo de metodologías de investigación - acción para la reconstrucción, que consideren las complejidades culturales, la creación de canales de comunicación e incidencia dentro de las colectividades y de estas con la sociedad y el desarrollo de procesos educativos vinculantes de pasado, presente y futuro. La mayoría de ellos, valga decir, utopías educativas (Freire, 1978; Morin, 2005; Simões Rodrigues, 2007 y Vilar, 1997).

Empero, al considerar esta formación que se plantea, hay que tener en cuenta también que en el mundo indígena, el camino de la sabiduría considera tres grandes ciclos:

a. El Runa Yachay o ciclo de formación en las ciencias ancestrales, que comprende el nivel del aprender a - pensar - haciendo comunitariamente y parte del nivel del aprender $-\mathrm{a}-$ aprender.

b. El Shuktak Yachay o ciclo de las ciencias occidentales, que abarca el nivel del aprender - a - aprender y parte del nivel del aprender $-a-$ desaprender $-y$ - reaprender

c. El Yachaypura o ciclo de la interculturalidad, que toma en cuenta parte del nivel del aprender - a
- desaprender - reaprender haciendo comunitariamente y el nivel del aprender - a - emprender haciendo comunitariamente (Milla Villena, 1983 y Viteri, 2003).

Considerado esto, es menester revisar también que el camino propuesto por los Kichwas de la Amazonía Ecuatoriana prevé rupturas curriculares que se corresponden con algunos de los niveles de aprendizaje, los cuales permiten incorporar cambios y novedades en el proceso formativo, a fin de mantener viva la curiosidad, la capacidad de riesgo y el interés de los estudiantes. Estas etapas equivalen a mecanismos de salida que posibilitan a los participantes obtener diversos grados técnicos académicos de formación, lo cual da lugar a carreras intermedias (Barrera, 2001; Calderón y Laserna, 1989; CONAIE, 1989; Karakras, 1990 y Wachtel, 1971).

Entonces, dado que los mundos indígenas tratan de resistir mediante la conservación de sus visiones de mundo (las cuales no toman en cuenta certezas preconcebidas), se está conociendo nuevamente la realidad. Para el pueblo Kichwa de la Amazonía Ecuatoriana, este conocimiento llega desde el humano vinculado a una cosmovisión que busca su Ally Causay ${ }^{3}$ como una forma de conservar equilibrios dinámicos, al mismo tiempo que en el Tukuy Pacha ${ }^{4}$ rastrea el camino permanente de conocimiento y comprensión de los ciclos de la vida en el Mushuk Allpa ${ }^{5}$. Los conocimientos concretos no se tratan como certezas, pues tienen una configuración dinámica de lo que se cree que es la realidad. Entonces, considerando que el aprendizaje debe fortalecer concomitantemente las habilidades individuales para accionar, gestionar y teorizar, debe también estimularse la capacidad de visualizar los procesos de trabajo y repensar el futuro (Viteri, 2003).

Además, deben considerarse el rescate, la reconstrucción y la socialización de capacidades, conocimientos y tecnologías individuales y colectivas en procesos de refundación cultural y la asociación intercultural solidaria, en una experimentación cuidadosa que observe los límites de cada una de las culturas. Todos los actores sociales son importantes y su participación en la resistencia política y la reconstrucción intercultural son decisorias en los escenarios comunes. Por esto, la socialización de todos los tipos de conocimientos hacia todos los actores es un tema prioritario, donde no caben criterios de exclusión por nivel de educación formal, edad, sexo o posicionamientos políticos previos a la convivencia intercultural. Ambos planes de trabajo 
conformarían el núcleo central de la acción académica (Leal, 2007).

Así como en los procesos de formación - información, investigación participativa y comunicación intercultural, debe predominar el abordaje problemático - sistémico y que los estudiantes se formen en temas teóricos y prácticos relevantes para sus comunidades, también es importante priorizar el rescate de los conocimientos locales y otros apropiados a esas realidades. Desde este enfoque, diferentes conocimientos podrían ser rescatados, reconstruidos y legitimados durante el propio proceso de formación, y serían respaldados en su constitución por la investigación - acción durante pasantías en comunidad (Leal, 2007).

A pesar de todo, la interculturalidad, entendida como proceso cognoscitivo y educativo, todavía es un tema poco trabajado en el ámbito de la educación superior latinoamericana, por ello solo podrá ser desarrollada en medio desde la experimentación y de la vivencia de la utopía. Empero, se podrían avizorar dos modalidades educativas básicas apropiadas a las condiciones comunitarias. En este entorno, la "Escuela para todos", viene a convertirse en un proceso de diálogo y formación continua de mujeres, jóvenes, adultos y niños sin las condiciones oficiales para ingresar a una universidad, pero con los intereses en adquirir una educación formal de larga duración. Por su parte, la "Universidad intercultural" se vuelve en un proceso de educación superior oficial para formar a jóvenes, en áreas afines al "Buen vivir". La "Escuela para todos" respondería así a los intereses colectivos y abrigaría una variedad de temas teóricos y prácticos determinantes para el fortalecimiento de las comunidades (Leal, 2007).

Entonces, el proceso formativo se establecería con base en los siguientes ejes de conocimiento:

a. Vida y expresiones culturales (cosmovisiones, discurso, lengua y sociedad).

b. Contextos (política y economía).

c. Agricultura sostenible (agroecología y tecnologías).

d. Equilibrios dinámicos (salud y sostenibilidad).

Como centros de apoyo e investigación, se podría pensar en un "Centro de Desarrollo Pedagógico Intercultural", que apoyaría las actividades pedagógicas y refinaría enfoques metodológicos y temáticos; el "Centro de Salvamento de Conocimientos", que trabajaría directamente con los docentes y estudiantes en activi-
Figura 3

El camino del filósofo (Yachak ${ }^{6}$ ) del Buen Vivir Indígena

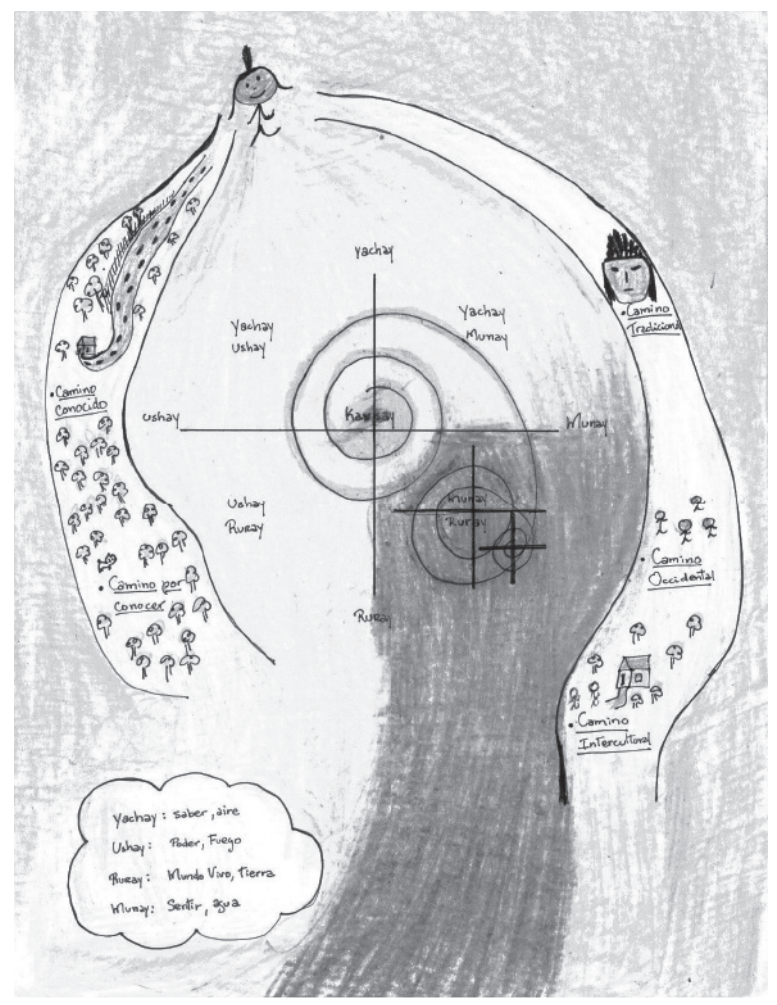

Fuente: Leal (2007). "Diario de campo núm. 4"

dades de investigación, recuperación y sistematización de conocimientos, en todas las áreas relevantes para la sostenibilidad; el "Centro del Discurso Intercultural y Derechos Económicos, Sociales y Culturales", que realizaría el rescate de las lenguas locales comprendidas como condensadores culturales e instrumentos para acción y el reconocimiento y manejo de las herramientas DESC y el "Centro de Desarrollo Tecnológico", con el cual se rescatarían, reconstruirían y sistematizarían tecnologías y fusiones tecnológicas interculturales y ejecutaría proyectos de investigación en comunidades, articulando en ello a los estudiantes (Leal, 2007).

\section{REFLEXIONES FINALES Y RECOMENDACIONES}

El Nuevo Humanismo procura generar espacios crecientes de libertad, bienestar y participación democrática y, junto con los paradigmas occidentales, emergen otros nuevos, que condensan el pensamiento ancestral y moderno y que no traen recetas estáticas o genéricas apegadas a métodos pre-existentes. Las nuevas concepciones afloran y se estructuran en su 


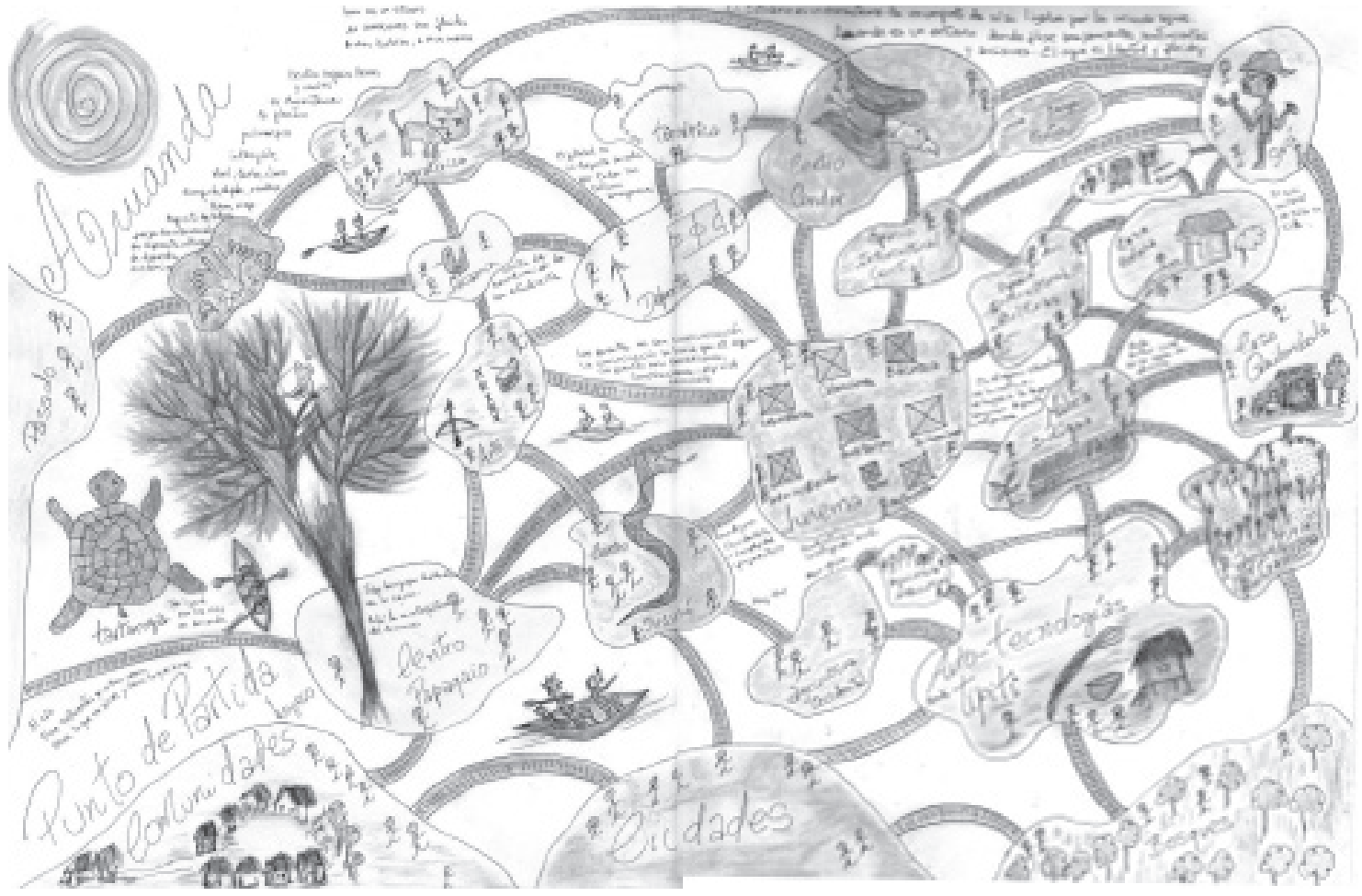

Fuente: Leal (2007). "Diario de campo núm. 4"

propia informalidad. Es la emergencia de una ciencia del pueblo, no mercantil ni escolástica, sino solidaria y orgánicamente constituida para resolver los obstáculos en la localidad. Nace con procesos de autoconocimiento, forjados entre actores y movimientos sociales, y también en los ambientes de los institutos de investigación y de cooperación internacional, frente al hecho ineludible de desarrollar herramientas de análisis más afines a la realidad local "para provocar y apoyar cambios estructurales y puntuales". Es así como los pueblos indígenas, más que en un caso interesante de estudio académico, se convierten en una reflexión existencial que cuestiona nuestra forma de vivir y convivir con nuestra especie y con el planeta y además, nos brindan perspectivas y soluciones teóricas y prácticas para la resolución de conflictos y necesidades emergentes, expresados en las enseñanzas de sus historias, ritualidad y sistemas adaptativos o tecnología locales. En los inicios del siglo XXI, humanistas y científicos sociales revalorizaron los saberes y cosmovisiones locales y les brindaron un mayor reconocimiento, local, nacional e incluso internacional; gracias a esto, incursionaron bravamente en estas densas realidades, traduciendo significados, comparando sistemas y "educando" profesionales indígenas, con una cosecha que hoy da frutos en diferentes países. Entonces, desde una perspectiva histórica, este rescate nos hace "redescubrirnos" como latinoamericanos e incluso nos ayuda a repensar vías alternativas para la supervivencia futura en este continente.

Como recomendación metodológica al investigar sobre pueblos indígenas, se debe mencionar la necesidad de explorar la mayor cantidad posible de fuentes documentales y ante todo, llevar a cabo visitas a la zona de estudio. Muchas de las investigaciones existentes (y a las que se tiene mayor acceso) han sido realizadas por personas ajenas a las culturas, por lo que sus hallazgos no están exentos de interpretaciones erróneas de hechos y sucesos históricos, caracterizados por una suerte de "subjetivismo cultural". Para solventar esta carencia, es necesario convivir con los descendientes vivos de la cultura ancestral. Como lo señala Hurtado (2010) la investigación debe ser significativa e impactante en el ámbito personal, porque se debe realizar de manera 
cercana, con las personas y grupos sociales protagonistas (a pesar de las limitaciones de tiempo y distancia).

Los investigadores sociales son sujetos externos que observan y por ende, sus percepciones y análisis están mediados por la experiencia de vida de cada individuo. Pero inevitablemente, de alguna manera se convierten también en actores al entrar en contacto e interactuar con los procesos y las personas que se estudian y hasta se ven impactados por ellos. Cuando el investigador opta por el trabajo de campo, solo se acerca a vivir parcial o temporalmente aquello que estudia, lo cual lo mantiene a distancia. Puede así analizar los procesos sin las ideas, los sentimientos y las pasiones propias de sus protagonistas. Empero, aquellos investigadores que intentan incursionar temporalmente en las vidas de otros, deben estar conscientes y alertas de sus limitaciones: aunque nunca dejará de ser un agente externo, tiene mayores posibilidades de sensibilizarse y de conocer más profundamente a través del contacto directo y las experiencias compartidas. Esta experiencia directa puede ser alimentada y fortalecida a través de las fuentes secundarias o indirectas.

Como consideración final, se debe recordar que, según Hurtado (2010) la investigación es una labor siempre inconclusa. Esto es especialmente válido en los procesos acelerados de cambio social. En ese sentido, lo que hasta ahora hemos podido conocer y analizar en estas investigaciones es solamente una parte de su vasta, compleja y cambiante realidad pues, la investigación solo capta un momento de algo que continúa desenvolviéndose y cambiando. Cada generación de la humanidad percibe de forma diversa su historia y su realidad y las convierte así en un objeto dinámico, fruto de la interacción entre el presente y el pasado. Este proceso es continuo y acelerado en virtud de los adelantos que aporta la tecnología y el desarrollo de la comunicación para la investigación científica. Cada día suceden nuevos descubrimientos, por lo que no pueden existir análisis o conclusiones definitivas. Solo pueden identificarse ciertas tendencias, lo que resulta evidente en el quehacer de las ciencias sociales como compromiso permanente de comprensión de realidades humanas en constantes e impredecibles cambios y transformaciones. Por lo tanto, es imposible pretender lo cierto y lo preciso en el conocimiento humano sobre la realidad social. Sin embargo, a pesar de estas limitaciones metodológicas, consideramos que se debe promover en las nuevas generaciones la realización de estudios concretos que rescaten el pensamiento original de las comunidades ancestrales de América Latina. Las culturas indígenas existentes en la actualidad y las que existieron, ofrecen una gran cantidad de casos interesantes para explorar. Por ello, con el insumo de mayores conocimientos será posible avanzar en la determinación de aportes al pensamiento universal. Estas investigaciones son un primer paso, pero pueden ser complementadas en el futuro, ya que constituyen una aproximación novedosa a un tema relativamente desconocido y un punto de partida para un conocimiento más comprensivo sobre la realidad de la región.

\section{NOTAS}

1 Como parte de las investigaciones realizadas, se destaca el trabajo de las académicas Leal y Vega. En primer lugar, la disertación doctoral de Leal (2007) examina la relación entre cuatro procesos emergentes de desarrollo: agricultores ecológicos organizados en Costa Rica, indios kuna en Panamá, Programa "Raízes" en Brasil y Movimiento Indígena Ecuatoriano. Ese estudio trata sobre la sostenibilidad de la vida y de las culturas, relacionando todo ello con la irrupción de una agricultura que rescata los conocimientos ancestrales y respeta los límites ecológicos de los sistemas productivos: esa es la agricultura para la vida. En segundo lugar, en la disertación doctoral de Vega (2013) se rescata la cosmovisión, la identidad de la cultura maya, sus valores de "conexión con la Madre Tierra", su responsabilidad social comunitaria y cómo estos elementos influyen en el desarrollo de prácticas ambientales tradicionales orientadas al logro del "Buen Vivir". El trabajo de campo realizado permite conocer cómo las prácticas ambientales del rescate alimenticio de la nuez de ramón y la aplicación de la medicina tradicional indígena, se pueden considerar ejemplos concretos de un pensamiento ambiental latinoamericano en acción, que ha sido reconocido con galardones internacionales de gran prestigio, al ser iniciativas comunitarias que rescatan conocimientos tradicionales y procuran una mejor calidad de vida mediante la conservación y el uso sostenible de la naturaleza.

2 En este caso, se considera "político" todo lo referente al medio de conquista de los espacios para el cambio.

3 Significa encuentro o equilibrio. La ontología es variable: no existen certezas, se intuye, se experimenta, no se concluye. El encuentro y el equilibrio tienen cauces naturales. Es el sentir la plenitud de la vida en todos los eventos, que pueden ser percibidos como circunstanciales, aunque que estas circunstancias están dentro de los límites de una normalidad de eventos concretos, de realidades cuyos cambios son asimilados en el tiempo real de las culturas.

$4 \quad$ Es aprender y reaprender de la vida a cada momento. Se forman bancos de impresiones más tenues que las certezas occidentales. Nada es definitivo, se aprende con cada evento y los criterios y definiciones serán flexibles. Se mira de largo, de cerca, se 
Investigación humanística en América Latina: experiencias con comunidades indígenas

olfatea, se toca, se levanta, se experimenta, se asocia a las impresiones pasadas y se mira los resultados. Se deja o se toma la cosa y luego se piensa lo que era, a veces se cuenta a los demás la experiencia vivida.

5 Al mismo tiempo que nada es concreto, existe la certeza del ciclo, del regreso de los elementos y de los eventos. Se sabe que los días y lo que se verá en este día pueden repetirse, pero nunca hay certezas. Se olfatea el clima, los olores del bosque y de los animales. Se sabe qué comer y qué es remedio para que a partir de la observación de los eventos y otros organismos naturales se aprenda que son distintos.

6 El médico y el sabio, en el idioma Kichwa del Ecuador.

\section{REFERENCIAS BIBLIOGRÁFICAS}

Abarca, F. (2013). Cantos de amor y esperanza. Textos de apoyo conceptual para el taller: Fundamentos básicos de Mediación Pedagógica y la Metodología Participativa. Organización Internacional para las Migraciones (OIM). Sin publicar. San José. Costa Rica.

Abarca, F. (2012). Nuevas visiones para tiempos nuevos: Hacia aprendizajes holistas como derecho de la humanidad. Revista Latinoamericana de Derechos Humanos. 23 (2). Facultad de Filosofía y Letras de la Universidad Nacional de Costa Rica, 61-85.

ARUANDA. (2012). Informe DESC/Capítulo Costa Rica. (Leal Rodrigues, ed.). Centro de Estudios Generales, Universidad Nacional. Heredia, Costa Rica.

Aruanda. (2013). Proyecto Módulo de Formación en Estudios Humanísticos, Interculturalidad y Derechos Económicos, Sociales y Culturales de los Pueblos Indígenas-Red DESC-ARUANDA. (Leal Rodrigues, ed.). Centro de Estudios Generales, Universidad Nacional. Heredia, Costa Rica.

Barrera, A. (2001). Acción colectiva y crisis política: el movimiento indígena ecuatoriano en la década de los noventa. Quito: Ediciones Abya Yala.

Busch, L. y Sachs, C. (1981). The agricultural sciences and the modern world system. Science and agricultural development (Busch, L., ed.). New Jersey: Allanheld, Osmun \& Co. Publishers, Inc. Montclair.

Calderón, F. y R. Laserna. (1989). Nación, Estado y movimientos sociales regionales en Bolivia (1917-1983). Los movimientos populares en América Latina. Universidad de las Naciones Unidas: Siglo Veintiuno Editores.

CONAIE. (1989). Las nacionalidades indígenas en el Ecuador: Nuestro proceso organizativo. Quito: Ediciones Tincui - Abya Yala.

Fornet Betancourt, R. (2001). Transformación intercultural de la filosofía. Editorial Desclee De Brouwer: Bilbao, España.

Freire, P. (1978). Extensión e invasión cultural. In: ¿Extensión o comunicación? La concientización en el medio rural. Sibuna: México.
Hurtado, M. (2010). Petén: ¿la última frontera? La construcción social de una región. Disertación doctoral de la Universidad de Wageningen University, Países Bajos. Sin publicar.

Iglesias Díaz, C. (1999). Educar para la paz desde el conflicto. Alternativas teóricas y prácticas para la convivencia escolar. España: Ediciones HomoSapiens.

Karakras, A. (1990). Las nacionalidades indias y el estado ecuatoriano. Quito: Editorial Tincui - CONAIE.

Leal, D. y Vega. H. (2014). Humanidades e investigación: estrategias y metodologías de investigación-acción participativas en comunidades indígenas de América Latina. Ponencia presentada el IV Encuentro Latinoamericano de Metodología de las Ciencias Sociales (ELMECS). 27 - 29 de agosto. Universidad Nacional, Costa Rica.

Leal, D. (2007). Agricultura para la vida y reconstrucción de identidades: casos paradigmáticos en Costa Rica, Panamá, Brasil y Ecuador. Disertación doctoral en Estudios Latinoamericanos de la Universidad Nacional de Costa Rica. Sin publicar.

Leff, E. (1986). Ecología y capital: hacia una perspectiva ambiental del desarrollo. México, DF: Universidad Nacional Autónoma de México.

Leis, R. (1989). El Arco y la Flecha: apuntes sobre metodología y práctica transformadora. Programa Regional Coordinado de Educación Popular. Alforja, San José.

Milla Villena, C. (1983). Génesis de la cultura andina. Lima: Fondo Editorial C.A.P. Colección Bienal.

Morin, E. (2005). O método 3: conhecimento do conhecimento. Porto Alegre: Sulina.

Ribeiro, D. (1996). Os indios e a civilizacão. São Paulo: Companhia das Letras.

Simões Rodrigues, D. (2007). Educação, meio ambiente e cultura. Belém: UEPA.

Universidad Amawtay Wasi. (2001). Planes y programas del milenio. Quito: Facultad de Ciencias Agroecológicas.

Vega, H. (2013). Aportes al pensamiento latinoamericano desde la cosmovisión y las prácticas ambientales para el Buen Vivir: mujeres indígenas y campesinas en la Reserva de la Biósfera Maya. Disertación doctoral en Estudios Latinoamericanos de la Universidad Nacional. Costa Rica. Sin publicar.

Vilar, S. (1997). La nueva racionalidad: comprender la complejidad con métodos transdisciplinarios. Barcelona: Editorial Kairós.

Viteri, C. (2003). Visión indígena del desarrollo en la Amazonía. Modulo Amazonía contemporánea. Quito: Escuela de Derechos Indígenas, CDES.

Wachtel, N. (1971). Los vencidos: los indios del Perú frente a la conquista española (1530-1570). Madrid: Alianza Universidad. 